\title{
Temporal expectations modulate attentional capture
}

\author{
DOMINIQUE LAMY \\ Tel Aviv University, Tel Aviv, Israel
}

\begin{abstract}
Subjects' ability to override attentional capture by salient distractors during singleton search has been attributed to the adoption of an attentional set for the type of discontinuity characterizing the target (Folk, Remington, \& Johnston, 1992) or to fast disengagement of attention from the salient distractor's location (Theeuwes, Atchley, \& Kramer, 2000). The present results support an alternative account by showing that temporal expectancies modulate attentional capture. In a color singleton search, an irrelevant onset preceding the target by a given time interval produced capture when the distractor-to-target interval varied unpredictably but failed to do so when this interval was predictable. Moreover, with unpredictable intervals and moderately salient stimuli, capture was overridden at the expected average interval. These findings invite caution when stimulus onset asynchrony manipulations are used to study the temporal deployment of attention, since they demonstrate that such manipulations introduce powerful temporal expectations.
\end{abstract}

The extent to which top-down control may be exerted to prevent irrelevant salient objects from capturing attention is one of the most debated issues in attention research of the last decade or so (for reviews, see Rauschenberger, 2003; Ruz \& Lupiáñez, 2002). Early findings (e.g., Theeuwes, 1991) suggested that the most salient item captures attention during visual search, despite the observer's efforts to the contrary. Later studies (e.g., Bacon \& Egeth, 1994; Lamy \& Tsal, 1999; Yantis \& Egeth, 1999) identified important boundary conditions to such attentional capture, by showing that it occurs only when subjects adopt an attentional set for salient objects - that is, in cases in which the target itself is also a salient object (but see Theeuwes, 2004).

However, even during singleton search, attentional capture by irrelevant singletons is not the rule. A number of studies (e.g., Folk, Remington, \& Johnston, 1992; Folk, Remington, \& Wright, 1994; Pratt, Sekuler, \& McAuliffe, 2001) have suggested that subjects' attentional set can be further tuned to specific types of discontinuities. They have shown that, for instance, an irrelevant onset captures attention in search for an onset target, but not in search for a color singleton, and vice versa. It is noteworthy that in these studies, when the irrelevant singleton was defined by a different dimension than the target and, thus, produced no capture, it typically preceded the target by $150 \mathrm{msec}$. This observation led Theeuwes, Atchley, and Kramer (2000) to propose that the irrelevant singleton may grab attention early on but that subjects are able to

\footnotetext{
Support for this research was provided by the Israel Science Foundation, Grant 1382-04 to D.L. Correspondence concerning this article should be addressed to D. Lamy, Department of Psychology, Tel Aviv University, Ramat Aviv, P. O. Box 39040, Tel Aviv 69978, Israel (e-mail: domi@post.tau.ac.il).
}

overcome attentional capture by the time the target display is presented. This disengagement hypothesis was supported by the results of three experiments in which distractor-to-target stimulus onset asynchrony (SOA) was varied. Significant interference by the irrelevant singleton was observed at the 50- and 100-msec SOAs, but not at longer SOAs (for related findings, see Kim \& Cave, 1999; Lamy \& Egeth, 2003, Experiment 1).

This interpretation of the results relies on the widely accepted premise that manipulations of SOA provide a window into the time course of attentional deployment (e.g., Kim \& Cave, 1999; Klein, 2000; Posner \& Cohen, 1984; Yantis \& Jonides, 1990). However, SOA manipulations can also affect processing by creating expectations about the moment in time at which the target will appear. Indeed, several studies have demonstrated subjects' ability to orient attention in time (e.g., Coull, Frith, Büchel, \& Nobre, 2000; Miniussi, Wilding, Coull, \& Nobre, 1999). On the basis of the warning signal literature (e.g., Niemi \& Näätänen, 1981), these authors reported that subjects can prepare for the time of target onset and will show a cost when temporal expectancies are violated.

The hypothesis suggested here is that temporal expectations might also modulate subjects' ability to overcome attentional capture. That is, it might be easier to override attentional capture when the interval of time during which an attentional shift toward the salient distractor must be withheld (distractor-to-target SOA) is predictable. Two important observations emerge from a reexamination of the literature with this hypothesis in mind.

First, in tasks that involved searching for a known singleton while ignoring a different singleton distractor, capture effects were typically observed when the distractorto-target SOA was not predictable (e.g., Folk et al., 1992, Experiment 4; Lamy \& Egeth, 2003, Experiments 1-3; Theeuwes et al., 2000), but not when it was fixed and, 
thus, known in advance (e.g., Folk \& Remington, 1998; Folk et al., 1992, Experiments 1-3; Pratt et al., 2001).

Second, when recovery from capture was observed, evidence for capture sometimes reappeared at the longest SOAs (see, e.g., Theeuwes et al., 2000, Experiments 3 and 4). Although this pattern of results cannot be accommodated by the disengagement hypothesis, it parallels the findings obtained in temporal cuing studies. In these studies, a warning cue typically instructs subjects to expect a target after either a short (e.g., $600 \mathrm{msec}$ ) or a long (e.g., $1,400 \mathrm{msec}$ ) interval of time. This warning cue is valid on most trials and invalid on a minority of trials. Validity effects were consistently reported for targets appearing after short intervals. That is, when the target appeared $600 \mathrm{msec}$ after the warning cue, subjects responded faster when they had expected the target at that time, rather than after a longer interval. In contrast, validity effects were smaller (e.g., Coull et al., 2000) or unreliable (Coull \& Nobre, 1998; Miniussi et al., 1999) for targets appearing after long intervals. That is, when the target appeared $1,400 \mathrm{msec}$ after the warning cue, subjects did not always respond more quickly when they had expected the target at that time rather than after a shorter interval.

In the context of attentional capture, I suggest a temporal expectation account that can accommodate these observations. According to this account, (1) knowing how long after distractor onset the target will appear facilitates resistance to capture. This explains why capture effects are typically observed with variable, but not with fixed, distractor-to-target intervals. (2) When distractorto-target SOAs vary from trial to trial, subjects prepare for the average expected interval. This explains why capture effects are typically observed with shorter-than-average intervals, disappear at the average expected interval, and only sometimes reemerge after this interval has elapsed.

In Experiment 1, the temporal expectation hypothesis was tested directly by comparing capture at various SOAs when these were presented in different homogeneous blocks relative to when they were randomly mixed within blocks. The stimuli and procedure were chosen to be as close as possible to Folk et al.'s (1992). The subjects searched for a target defined by its known unique color, while ignoring an irrelevant onset that preceded the target by an SOA of 50,175 , or $300 \mathrm{msec}$, the location of which was uncorrelated with the location of the target. Spatial capture was measured as a performance facilitation when the target appeared at the same location as the irrelevant onset, relative to when it appeared at a different location. According to the temporal expectation hypothesis suggested here, (1) capture effects should be larger in the mixed-SOAs than in the fixed-SOA condition, and (2) in the mixed-SOAs condition, capture should be lowest at the average expected SOA - that is, after a $175-\mathrm{msec}$ interval. According to Folk et al.'s (1992) contingent capture account, no capture should be observed in either the mixed-SOAs or the fixed-SOA condition, because the distractor and the target are defined along different stimulus dimensions. Finally, Theeuwes et al.'s (2000) attentional disengagement account predicts capture at the early SOA and recovery later on, irrespective of whether SOAs are mixed or blocked.

\section{EXPERIMENT 1}

\section{Method}

Subjects. The subjects were 18 Tel Aviv University undergraduate students, who participated in the experiment for course credit. All reported having normal or corrected-to-normal visual acuity and normal color vision.

Apparatus. The same apparatus was used in all the experiments. Displays were generated by an Intel Pentium 4 computer attached to a 15 -in. TFT monitor, using $640 \times 480$ resolution graphics mode. Responses were collected via the computer keyboard. A chinrest was used to set viewing distance at $50 \mathrm{~cm}$ from the monitor.

Stimuli. The fixation display was a gray + sign in the center of a black background. The ready display consisted of the fixation display, on which four gray outline peripheral boxes $\left(1.15^{\circ} \times 1.15^{\circ}\right)$ placed $4.7^{\circ}$ above, below, to the left, and to the right of fixation were superimposed. The distractor display consisted of the ready display with a set of four small gray circles $\left(0.36^{\circ}\right.$ in diameter $)$ in a diamond configuration, surrounding one of the peripheral boxes at an approximate distance of $0.3^{\circ}$. The interstimulus display was identical to the ready display. The target display consisted of the ready display with the addition of two $\mathrm{x}$ and two $=\operatorname{signs}\left(0.57^{\circ}\right.$ of visual angle $)$ randomly assigned to each of the boxes. Three signs were gray, and the remaining sign was red. Thus, each target display contained one color singleton, which was the target. The red and gray colors were matched for luminance, using a light meter (Minolta ColorCAL colorimeter, Cambridge Research Systems).

Procedure. The fixation display appeared for $500 \mathrm{msec}$ and was replaced by the distractor display, which appeared for $50 \mathrm{msec}$. The interstimulus display followed for a variable duration, producing three possible distractor-to-target display SOAs: 50, 175, or $300 \mathrm{msec}$. The target display remained visible until the subjects responded or 2,000 msec had elapsed. The screen went blank for $500 \mathrm{msec}$ before the next trial began.

The subjects indicated what sign had appeared within the unique red square in the target display. They pressed 3 if it had been $x$ and $\mathrm{z}$ if it had been $=$. They were asked to respond as fast as possible while keeping the number of errors at a minimal level. Error trials were followed by a 500-msec feedback beep. Eye movements were not monitored, but the subjects were explicitly requested to maintain fixation throughout each trial.

Design. The design included three within-subjects factors (SOA $[50,175$, or $300 \mathrm{msec}$ ], distractor-target location [same vs. different location], and block type [fixed SOA and mixed SOAs]) and two between-subjects factors (order of block type and order of SOA blocks in the fixed-SOA condition). The subjects were randomly assigned to each combination of the between-subjects variables.

In the fixed-SOA condition, the distractor-to-target SOA remained constant for an entire block of trials. There were three blocks of 140 trials each in the fixed-SOA condition, one for each possible SOA. In the mixed-SOAs condition, the three possible SOAs were equally probable and randomly mixed within each block of trials. There were three blocks of 120 trials each in the mixed-SOAs condition, which differed only in trial order. On same-location trials, the abrupt-onset distracting dots appeared at the same location as the target, whereas on different-location trials, the distractor and the target occupied different positions. Conditions of distractor-target location were randomly intermixed within each block of experimental trials. Each block was preceded by 20 practice trials. Thus, the experiment included 80 practice trials and 720 experimental trials. Except for the first 20, the practice trials appeared to be part of the experimental blocks, so that the subjects were not aware of the transitions from one condition to the next. 


\section{Results and Discussion}

Mean reaction times (RTs) on correct trials and mean error rates are presented in Figure 1. In all the RT analyses, error trials ( $4.8 \%$ of all the trials) were removed from analysis, and RTs for each subject were sorted into cells according to the conditions of SOA, distractor-target location, and block type. RTs exceeding the mean of a given cell by more than 3.5 standard deviations (fewer than $0.5 \%$ of all the observations) were trimmed. Preliminary analyses revealed no significant effect involving SOA block order or block type order. Therefore, the data were collapsed across these variables.

Reaction times. An ANOVA with SOA, distractortarget location, and block type as factors revealed a significant main effect of SOA $[F(2,34)=6.67, p<.004]$, with shorter RTs on 175- and 300-msec SOA trials than on 50-msec trials. Planned comparisons revealed that the location effect was significant in the mixed-SOAs condition $[F(1,17)=5.31, p<.04]$, but not in the fixed-SOA condition $(F<1)$. However, these effects were qualified by a significant triple interaction between SOA, distractortarget location, and block type $[F(2,34)=6.63, p<.004]$. Paired comparisons showed that in the mixed-SOAs condition, the distractor-target location effect was significant for the 50- and 300-msec SOAs $[F(1,17)=7.04, p<.02$, and $F(1,17)=6.53, p<.03$, respectively], but not for the 175 -msec SOA $[F(1,17)=1.94, p>.1]$, whereas in the fixed-SOA condition, this effect was nonsignificant across SOAs $[F<1, F(1,17)=2.97, p>.1$, and $F<1$, for the 50-, 175-, and 300-msec SOAs, respectively].

Accuracy. The main effect of SOA was significant $[F(2,34)=4.05, p<.03]$, with fewer errors on 175 - and 300 -msec SOA trials than on 50-msec trials. Only the interaction between distractor location and SOA approached significance $[F(2,34)=3.24, p<.06]$. Paired comparisons revealed that error rates were actually higher in the same-location than in the different-location condition at the 50 -msec SOA $[F(1,17)=5.27, p<.03]$, with no difference between the two location conditions at the 175 - or $300-\mathrm{msec}$ SOAs $[F(1,17)=1.46, p>.2$, and $F<1$, respectively].

The results supported the hypothesis that temporal expectations modulate attentional capture. When the distractor-totarget SOA was predictable (fixed-SOA condition), the irrelevant onset produced no observable capture of attention at any of the SOAs tested. When distractor-to-target SOA varied randomly (mixed-SOAs condition), spatial capture by the same distractor was significant for all SOAs except for the average expected SOA, although it should be noted that a speed-accuracy trade-off was apparent at the 50-msec SOA.

The 175 -msec SOA was the average expected SOA in the present experiment, but it is also close to the prototypi-
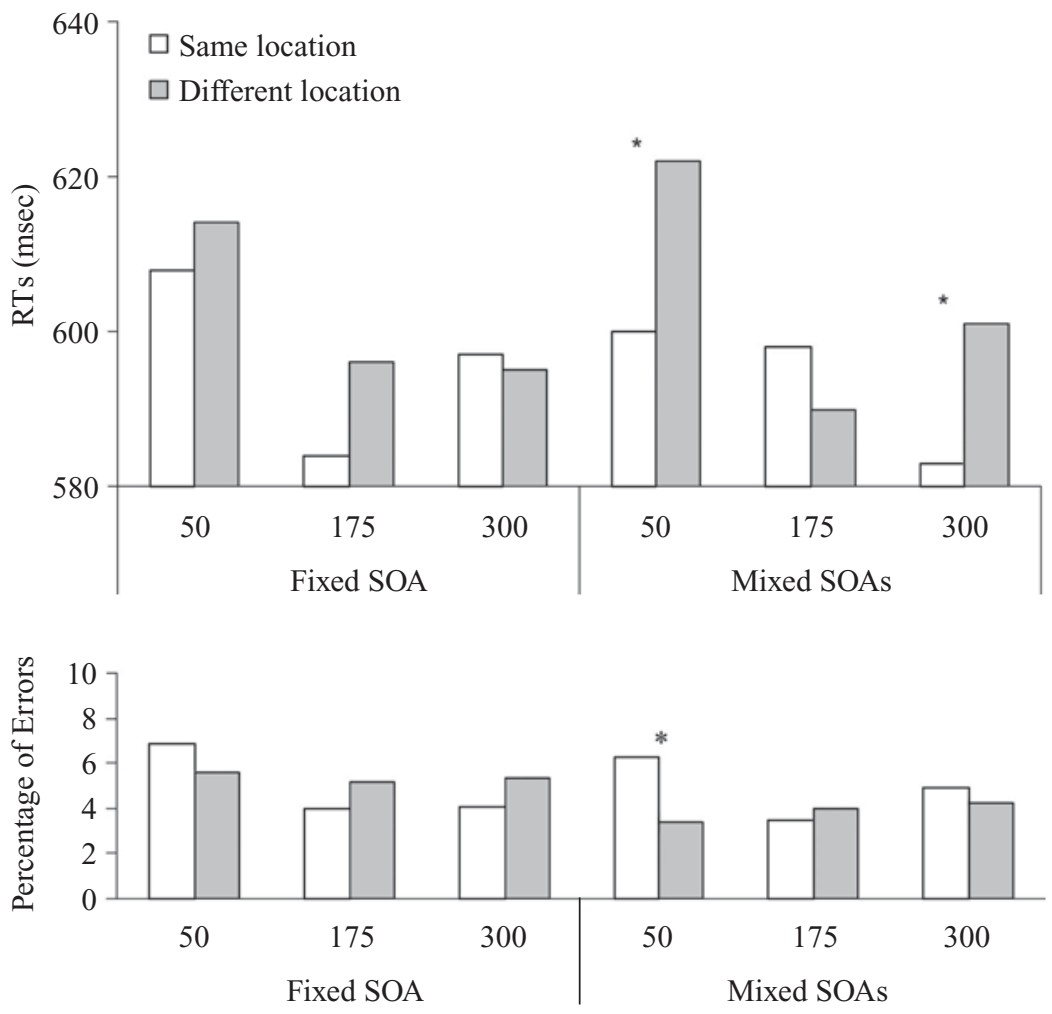

Figure 1.Target identification performance as a function of distractor-target location (same vs. different), distractor-to-target stimulus onset asynchrony (SOA, in milliseconds), and block type (fixed SOA vs. mixed SOAs) in Experiment 1. Upper panel: mean reaction times (RTs) in milliseconds. Lower panel: percentage correct. ${ }^{*} p<.05$. 
cal SOA for which no capture has been observed in earlier studies (e.g., Folk et al., 1992; Lamy \& Egeth, 2003; Pratt et al., 2001; Theeuwes et al., 2000). Thus, it is possible that for reasons yet to be clarified, resistance to capture is optimal when the target follows the distracting event by roughly $150 \mathrm{msec}$, irrespective of whether this is the average expected interval. The objective of Experiment 2 was to test this alternative account.

\section{EXPERIMENT 2}

This experiment was identical to Experiment 1, except that the SOAs were 175, 300, and $425 \mathrm{msec}$, instead of 50, 175, and $300 \mathrm{msec}$. Again, capture effects were expected to be larger in the mixed-SOAs condition than in the fixedSOA condition. Critically, in the mixed-SOAs condition, if subjects indeed prepare for the average distractor-totarget SOA, capture effects should be large at the 175- and 425-msec SOAs and minimal at the 300-msec SOA.

\section{Method}

Subjects. The subjects were 15 Tel Aviv University undergraduate students, who participated in the experiment for course credit. All reported having normal or corrected-to-normal visual acuity and normal color vision.

Apparatus, Stimuli, Procedure, and Design. The apparatus, stimuli, procedure, and design were the same as those in Experi- ment 1, except that the SOAs were 175, 300, and $425 \mathrm{msec}$, instead of 50,175 , and $300 \mathrm{msec}$.

\section{Results and Discussion}

Mean correct RTs and mean error rates are presented in Figure 2 . In all the RT analyses, error trials $(4.7 \%$ of all the trials) and outliers (following the same procedure as that in Experiment 1) were removed from analysis. Preliminary analyses revealed no effect involving SOA block order or block type order. Therefore, the data were collapsed across these variables.

Reaction times. The main effect of distractortarget location was significant $[F(1,14)=6.58, p<.03]$. Planned comparisons revealed that the location effect was significant in the mixed-SOAs condition $[F(1,14)=$ $12.62, p<.004]$, but not in the fixed-SOA condition $(F<1)$. However, these effects were again qualified by a significant triple interaction between SOA, distractortarget location, and block type $[F(2,28)=3.41, p<.05]$. Paired comparisons showed that in the mixed-SOAs condition, the distractor-target location effect was significant for the 175- and 425-msec SOAs $[F(1,14)=9.81, p<$ .008 , and $F(1,14)=9.44, p<.009$, respectively], but not for the $300-$ msec SOA $[F(1,14)=2.16, p>.1]$, whereas in the fixed-SOA condition, this effect was nonsignificant across SOAs $[F(1,14)=2.02, p>.1, F(1,14)=1.54$,
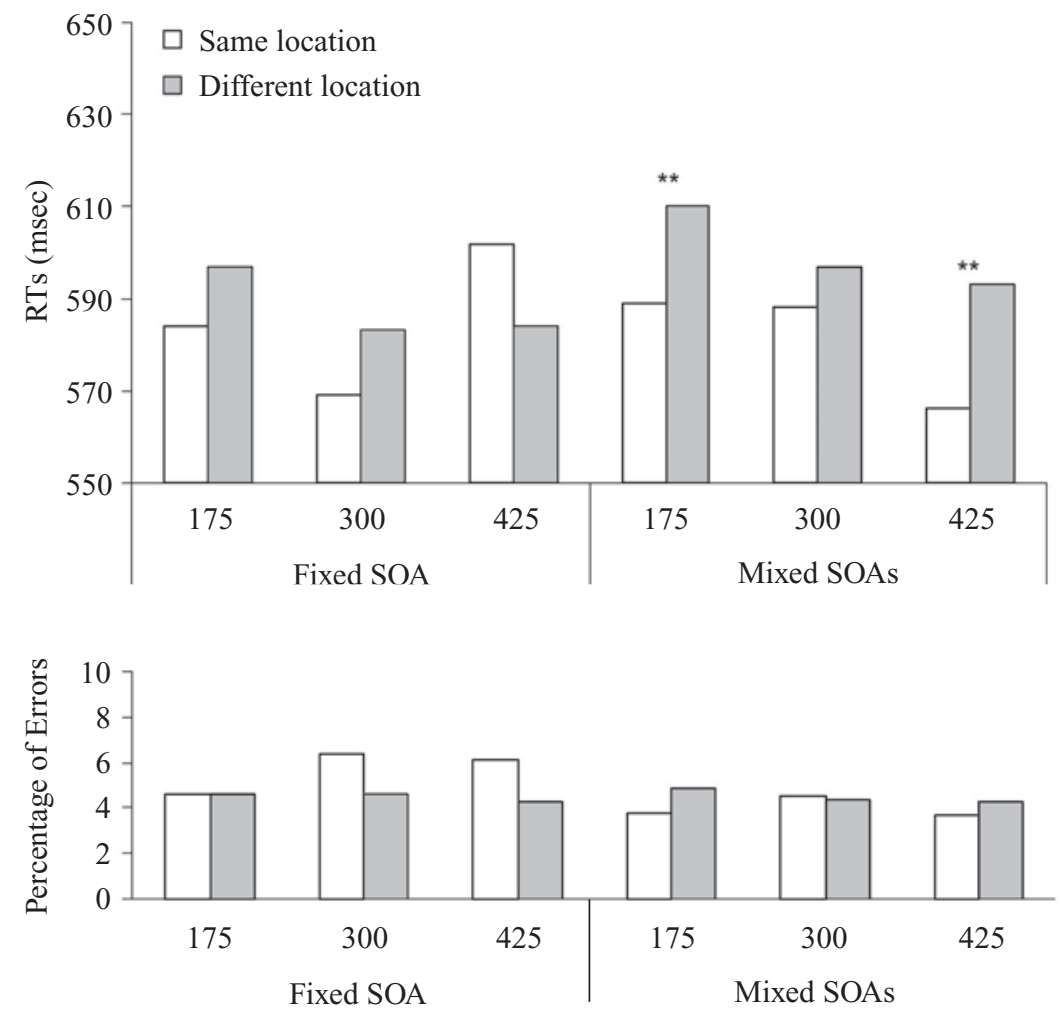

Figure 2. Target identification performance as a function of distractor-target location (same vs. different), distractor-to-target stimulus onset asynchrony (SOA, in milliseconds), and block type (fixed SOA vs. mixed SOAs) in Experiment 2. Upper panel: mean reaction times (RTs) in milliseconds. Lower panel: percentage correct. ${ }^{* *} p<.01$. 
$p>.2$, and $F(1,14)=2.94, p>.1$, for the 175-, 300-, and 425 -msec SOAs, respectively].

Accuracy. No effect of accuracy approached significance.

The results closely replicated the findings in Experiment 1 and thus supported the temporal expectation account. The temporal context in the present experiment produced dramatically different effects of the irrelevant onset at the 175- and 300-msec SOAs, relative to Experiment 1. These results are consistent with the idea that subjects prepare for the average expected SOA and argue against the notion that resistance to capture is optimal when the target follows the distracting event by $150-175 \mathrm{msec}$.

\section{EXPERIMENT 3}

The idea that temporal expectations help subjects overcome attentional capture by salient objects is inconsistent with the results of several studies in which the magnitude of attentional capture remained unchanged across all the SOAs tested (e.g., Folk et al., 1992, Experiment 4, Lamy $\&$ Egeth, 2003, Experiments 2 and 3). The fact that these studies did not differ from Experiments 2 and 3 in terms of the general procedure used suggests that stimulus factors may have created the observed discrepancy between the two sets of data. Accordingly, in Experiment 3, the possibility was tested that temporal expectations might not suffice to prevent attentional capture when the distracting events are strongly salient and might produce observable effects only with moderately salient stimuli.

In an effort to generalize the findings obtained in Experiments 1 and 2 to different stimuli, the materials used in Experiment 3 were similar to Lamy and Egeth's (2003, Experiment 2). The critical manipulation concerned the irrelevant onset's salience level.

\section{Method}

Subjects. The subjects were $15 \mathrm{Tel}$ Aviv University undergraduates, who participated in the experiment for course credit. All reported having normal or corrected-to-normal visual acuity and normal color vision.

Stimuli. The preonset display consisted of six gray circles equally spaced along the circumference of an imaginary circle, centered at fixation. The distractor display was identical to the preonset display, except that a white outline diamond was superimposed on one of the circles, so that their centers overlapped. In the high-salience distractor condition, the outline diamond was drawn with a 3-pixelthick stroke, whereas in the low-salience distractor condition, it was drawn with a 1-pixel-thick stroke. The interstimulus display was identical to the preonset display - that is, the abruptly onset diamond was no longer present. In the target display, one gray circle turned to red, and the other circles turned to light gray. At a viewing distance of $60 \mathrm{~cm}$, the centers of the shapes were $4.08^{\circ}$ from fixation. The diamond was $2.76^{\circ}$ tall and $2.25^{\circ}$ wide. The circles had a diameter of $2.04^{\circ}$. The $\mathrm{x}$ sign subtended $0.75^{\circ} \times 0.75^{\circ}$, and the $=$ sign subtended $0.31^{\circ} \times 1.02^{\circ}$. Except for the abruptly onset diamond in the highsalience distractor condition, all the stimuli were drawn with a 1pixel stroke. Light gray and red were matched for luminance.

Procedure and Design. Figure 3 illustrates the sequence of displays. The procedure was similar to that in Experiment 2, except for the following changes. After $500 \mathrm{msec}$, the fixation display was replaced by the preonset display, which remained on the screen for $1,000 \mathrm{msec}$. The distractor display then appeared for $50 \mathrm{msec}$ and was replaced by the interstimulus display for a variable duration. The distractor-to-target SOA was randomly drawn from the following values: $50,100,150,200,250$, and $300 \mathrm{msec}$. Thus, there was no fixed-SOA condition; that is, this experiment included only the mixed-SOAs condition. The design included three within-subjects factors: SOA, distractor-target location, and distractor salience (high vs. low salience). All the conditions were randomly mixed within blocks. Each subject performed 20 practice trials, followed by 720 experimental trials divided into six blocks of 120 trials each.

\section{Results and Discussion}

Mean correct RTs and mean error rates are presented in Figure 4. In all the RT analyses, error trials (3.4\% of all the trials) and outliers (same procedure as that in Experiment 1) were removed from analysis.

Reaction times. An ANOVA with distractor-target location, SOA, and distractor salience as factors revealed a main effect of distractor-target location $[F(1,28)=$ $106.54, p<.0001]$, with shorter RTs on same-location than on different-location trials. The main effect of SOA was also significant $[F(5,75)=3.10, p<.02]$, with a general tendency for RTs to go down as SOAs increased. The interaction between distractor-target location and distractor brightness was significant $[F(1,14)=12.22$, $p<.004]$, with a larger location effect for the highsalience than for the low-salience distractor. Planned comparisons revealed that the location effect was significant across SOAs in the high-salience condition $[F(1,14)=40.63, p<$ $.0001, F(1,14)=30.93, p<.0001, F(1,14)=6.56, p<.03$,
Fixation (500 msec)

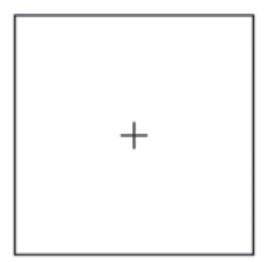

Distractor display (50 msec)
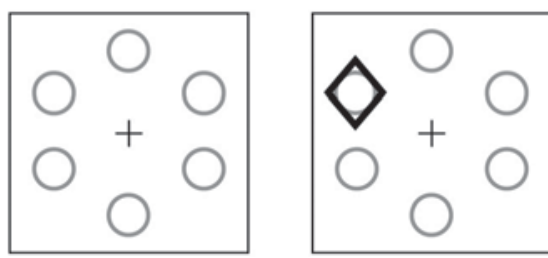

Interstimulus display (0 to $250 \mathrm{msec})$

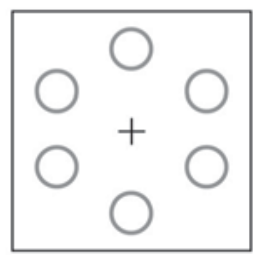

Target display (until response)

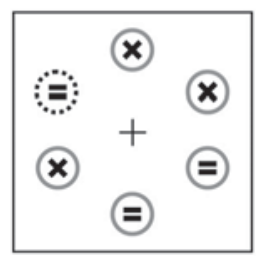

Figure 3. Stimuli and sequence of events in Experiment 3. The example corresponds to the same-location high-salience distractor condition. The dotted line in the figure was red, the gray lines were light gray, and the black lines were white. All the stimuli were presented against a black background. 

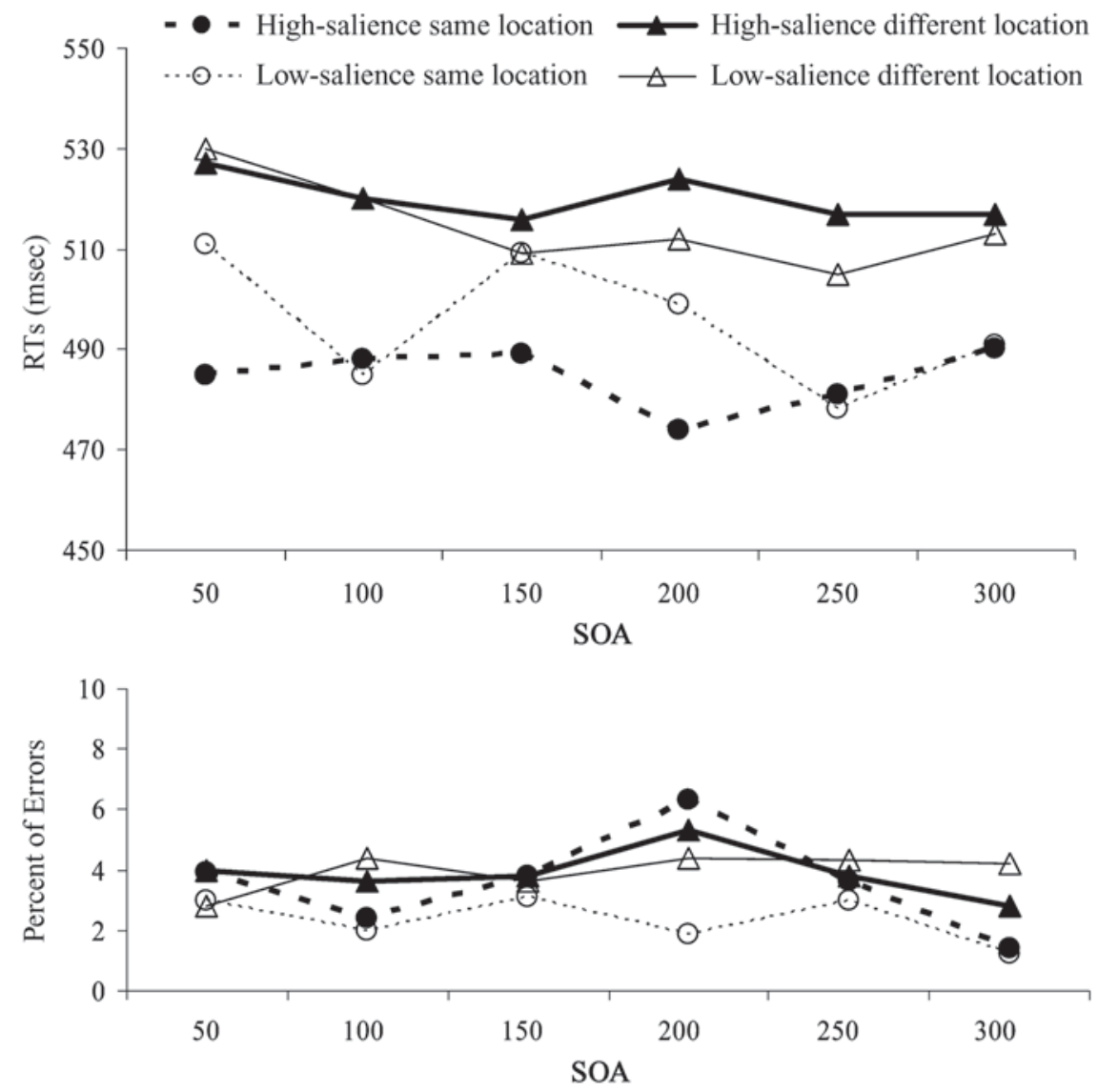

Figure 4. Target identification performance as a function of distractor-target location (same vs. different), distractor-to-target stimulus onset asynchrony (SOA), and distractor salience (low vs. high) in Experiment 3. Upper panel: mean reaction times (RTs) in milliseconds. Lower panel: percentage correct.

$F(1,14)=56.01, p<.0001, F(1,14)=14.76, p<.02$, and $F(1,14)=8.45, p<.02$, on 50-, 100-, 150-, 200-, 250-, and $300-\mathrm{msec}$ SOA trials, respectively]. In contrast, the same- vs. different-location effect was significant only for the short SOAs $[F(1,14)=7.18, p<.02$, and $F(1,14)=$ $30.33, p<.0001$, for 50- and 100-msec SOA trials, respectively] and for the long SOAs $[F(1,14)=7.45, p<$ .02 , and $F(1,14)=13.33, p<.003$, for 250 - and $300-$ msec SOA trials, respectively] and was nonsignificant for the intermediate SOAs $[F<1$ and $F(1,14)=1.83, p>$ .19 , for 150 - and 200-msec SOA trials, respectively].

Accuracy. Only the main effect of distractor-target location was significant $[F(1,14)=7.42, p<.02]$, with higher accuracy on same-location than on different-location trials.

The high-salience distractor produced capture effects across SOAs. The low-salience distractor produced capture effects for all the SOAs except for the intermediate SOAs - that is, around the average expected distractor-totarget interval. These results suggest that stimulus differences might account for the finding that preparation for the average expected SOA modulated attentional capture in some studies, but not in others.

\section{GENERAL DISCUSSION}

An irrelevant onset produced no observable capture with fixed distractor-to-target SOAs. In contrast, with unpredictable SOAs, spatial capture by the same irrelevant onset was significant for all the SOAs except for the average expected SOA. The present pattern of results suggests that temporal expectancies can strongly affect the extent to which capture by salient distractors can be overridden. Thus, it extends the notion that attentional control settings modulate involuntary capture of attention (Folk et al., 1992) by suggesting that such settings include temporal expectations, in addition to stimulus features. However, by showing that temporal expectations cannot modulate attentional capture by highly salient distractors, the present results also indicate that stimulus factors constrain the effects of attentional settings. In line with the ideas that I have presented elsewhere (Lamy, Carmel, Egeth, \& Leber, in press; Lamy, Leber, \& Egeth, 2004), such findings argue against the notion that one class of factors, either stimulus driven (e.g., Theeuwes, 2004) or goal oriented (e.g., Folk \& Remington, 1998), dominates attentional allocation. 
Only one other study (Milliken, Lupiáñez, Roberts, \& Stevanovski, 2003) has been done to investigate the interaction between temporal expectancies and attentional capture. Milliken et al. used uninformative peripheral spatial cues and showed that when the cue preceded the target by $100 \mathrm{msec}$, spatial cuing effects were larger when the subjects expected the cue-to-target SOA to be $100 \mathrm{msec}$ than when they expected it to be $900 \mathrm{msec}$. Thus, they observed maximal attentional capture, rather than optimal resistance to capture, at the expected SOA. Although the findings in the two studies appear to be inconsistent, an important difference between them may account for this discrepancy. Whereas in the present study, the distractor and the target were of a different discontinuity type (onset distractor and color target), they were of the same type in Milliken et al.'s study (onset cue and onset target). Previous studies (e.g., Folk et al., 1992; Lamy et al., 2004) showed that in the latter case, capture is observed. It is thus reasonable to suggest that in Milliken et al.'s study, the processes underlying resistance to capture were not triggered and could, therefore, not benefit from temporal expectancies. Instead, the cue produced the strongest effects when the subjects were in an optimal state of preparation for the target. To examine this claim further, the effects of temporal expectations with salient distractors that either match or do not match current attentional settings should be examined.

From a methodological viewpoint, the present findings invite caution when SOA manipulations are used to study the temporal deployment of attention, since they demonstrate that such manipulations introduce powerful temporal expectations.

From a theoretical viewpoint, although the present results clearly indicate that temporal expectations modulate the manifestation of capture, it is not entirely clear, at this point, what the underlying mechanisms might be. Note that the temporal expectations that differed across the relevant studies in the literature and thus were manipulated here concerned the interval between the appearance of the distractor and that of the target, rather than the time at which the distractor appeared. Indeed, the distractor always appeared at a fixed interval after the fixation display, so that preparation for the distractor's occurrence did not differ between conditions. How, then, might knowledge of the distractor-to-target interval affect attentional capture by that distractor? One possibility might be that, in line with Theeuwes et al.'s (2000) disengagement hypothesis, capture occurs regardless of temporal expectations but recovery from capture is most efficient when the target appears at an expected time than when it appears at an unexpected time. ${ }^{1}$ This account is consistent with the finding of capture in the mixed-SOAs condition and of no capture in the fixed-SOA condition. However, it cannot accommodate the specific pattern of results observed in the mixedSOAs condition in all three experiments - namely, the finding that capture disappears at the expected SOA but reappears thereafter. If the observers had disengaged their attention by, say, $175 \mathrm{msec}$ (Experiment 1), why would attention return to the distractor's location at longer SOAs?
One would have to postulate a reverse inhibition of return mechanism by which attention automatically returns to previously visited locations.

An alternative possibility might be that observers are able to prepare for withholding a shift of attention during a predefined interval of time. That is, when subjects expect a target to follow a salient event by a given amount of time, they may be best prepared to withhold an attentional shift to the salient event when their temporal expectations, whether implicit or explicit, are respected, because such temporal certainty will allow them to program the time window during which attentional shifts to irrelevant objects will be withheld. On the basis of the present findings, this time window appears to be rather short-perhaps, because it might be resource consuming. Such an account, however, is clearly speculative at this point and should be tested in further research.

\section{REFERENCES}

BACON, W. F., \& EGETh, H. E. (1994). Overriding stimulus-driven attentional capture. Perception \& Psychophysics, 55, 485-496.

Coull, J. T., Frith, C. D., Büchel, C., \& Nobre, A. C. (2000). Orienting attention in time: Behavioral and neuroanatomical distinction between exogenous and endogenous shifts. Neuropsychologia, 38, 808-819.

Coull, J. T., \& Nobre, A. C. (1998). Where and when to pay attention: The neural systems for directing attention to spatial locations and to time intervals as revealed by both PET and fMRI. Journal of Neuroscience, 18, 7426-7435.

FolK, C. L., \& Remington, R. [W.] (1998). Selectivity in distraction by irrelevant featural singletons: Evidence for two forms of attentional capture. Journal of Experimental Psychology: Human Perception \& Performance, 24, 847-858.

Folk, C. L., Remington, R. W., \& Johnston, J. C. (1992). Involuntary covert orienting is contingent on attentional control settings. Journal of Experimental Psychology: Human Perception \& Performance, 18, 1030-1044.

Folk, C. L., Remington, R. W., \& Wright, J. H. (1994). The structure of attentional control: Contingent attentional capture by apparent motion, abrupt onset, and color. Journal of Experimental Psychology: Human Perception \& Performance, 20, 317-329.

KIM, M.-S., \& CAVE, K. R. (1999). Top-down and bottom-up attentional control: On the nature of interference from a salient distractor. Perception \& Psychophysics, 61, 1009-1023.

KLEIN, R. M. (2000). Inhibition of return. Trends in Cognitive Sciences, 4, 138-147.

Lamy, D., Carmel, T., Egeth, H. E., \& Leber, A. B. (in press). Effects of search mode and intertrial priming on singleton search. Perception \& Psychophysics.

Lamy, D., \& Egeth, H. E. (2003). Attentional capture in singletondetection and feature-search modes. Journal of Experimental Psychology: Human Perception \& Performance, 29, 1003-1020.

Lamy, D., Leber, A., \& Egeth, H. E. (2004). Effects of task relevance and stimulus-driven salience within the feature search mode. Journal of Experimental Psychology: Human Perception \& Performance, 30, 1019-1031.

LAMY, D., \& TsaL, Y. (1999). A salient distractor does not disrupt conjunction search. Psychonomic Bulletin \& Review, 6, 93-98.

Milliken, B., Lupiáñez, J., Roberts, M., \& Stevanovski, B. (2003). Orienting in space and time: Joint contributions to exogenous spatial cuing effects. Psychonomic Bulletin \& Review, 10, 877-883.

Miniussi, C., Wilding, E. L., Coull, J. T., \& Nobre, A. C. (1999). Orienting attention in time: Modulation of brain potentials. Brain, 122, 1507-1518.

Niemi, P., \& NäÄTÄNEN, R. (1981). Foreperiod and simple reaction time. Psychological Bulletin, 89, 133-162.

Posner, M. I., \& CohEn, Y. (1984). Components of visual orienting. In 
H. Bouma \& D. G. Bouwhuis (Eds.), Attention and performance $X$ (pp. 531-556). Hillsdale, NJ: Erlbaum.

Pratt, J., Sekuler, A. B., \& McAuliffe, J. (2001). The role of attentional set on attentional cueing and inhibition of return. Visual Cognition, 8, 33-46.

Rauschenberger, R. (2003). Attentional capture by auto- and allocues. Psychonomic Bulletin \& Review, 10, 814-842.

Ruz, M., \& LuPiáñEZ, J. (2002). A review of attentional capture: On its automaticity and sensitivity to endogenous control. Psicológica, 23, 283-309.

Theeuwes, J. (1991). Cross-dimensional perceptual selectivity. Perception \& Psychophysics, 50, 184-193.

THEeUwEs, J. (2004). Top-down search strategies cannot override attentional capture. Psychonomic Bulletin \& Review, 11, 65-70.

Theeuwes, J., Atchley, P., \& Kramer, A. F. (2000). On the time course of top-down and bottom-up control of visual attention. In S. Monsell
\& J. Driver (Eds.), Attention and performance XVIII: Control of cognitive processes (pp. 105-124). Cambridge, MA: MIT Press.

Yantis, S., \& EgEth, H. E. (1999). On the distinction between visual salience and stimulus-driven attentional capture. Journal of Experimental Psychology: Human Perception \& Performance, 25, 661-676.

Yantis, S., \& Jonides, J. (1990). Abrupt visual onsets and selective attention: Voluntary versus automatic allocation. Journal of Experimental Psychology: Human Perception \& Performance, 16, 121-134.

\section{NOTE}

1. I thank Brad Gibson for this suggestion.

(Manuscript received November 18, 2004; revision accepted for publication April 27, 2005.) 